Original article

\title{
Elevated expression levels of lysyl oxidases protect against aortic aneurysm progression in Marfan syndrome
}

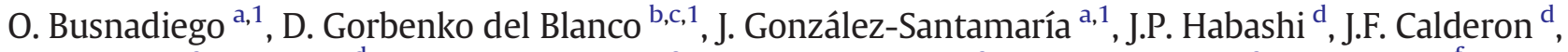 \\ P. Sandoval a, D. Bedja ${ }^{\text {d }}$, J. Guinea-Viniegra ${ }^{\text {e }}$, M. Lopez-Cabrera ${ }^{\text {a }}$, T. Rosell-Garcia ${ }^{\text {a }}$, J.M. Snabel ${ }^{\mathrm{f}}$, \\ R. Hanemaaijer ${ }^{f}$, A. Forteza ${ }^{\text {g }}$, H.C. Dietz ${ }^{\text {d }}$, G. Egea ${ }^{\text {b,c,2 }}$, F. Rodriguez-Pascual ${ }^{\mathrm{a}, *, 2}$
}

${ }^{a}$ Centro de Biología Molecular "Severo Ochoa" Consejo Superior de Investigaciones Científicas (C.S.I.C.)/Universidad Autónoma de Madrid (Madrid), Madrid, Spain

bepartamento de Biología Celular, Inmunología y Neurociencias, Facultad de Medicina, Universidad de Barcelona, Barcelona, Spain

c Institut d'Investigacions Biomèdiques August Pi i Sunyer (IDIBAPS), Barcelona, Spain

d Institute of Genetic Medicine, Johns Hopkins University School of Medicine, Baltimore, MD, USA

e Fundación Banco Bilbao Vizcaya-CNIO Cancer Cell Biology Program, Centro Nacional de Investigaciones Oncológicas, Madrid, Spain

${ }^{\mathrm{f}}$ TNO Metabolic Health Research, Leiden, The Netherlands

${ }^{\mathrm{g}}$ Hospital Universitario 12 de Octubre, Unidad de Marfan, Madrid, Spain

\section{A R T I C L E I N F O}

\section{Article history:}

Received 2 October 2014

Received in revised form 28 April 2015

Accepted 11 May 2015

Available online 16 May 2015

\section{Keywords:}

Lysyl oxidases

Extracellular matrix

Aneurysm

Marfan syndrome

Collagen

Transforming growth factor- $\beta$

\begin{abstract}
A B S T R A C T
Patients with Marfan syndrome (MFS) are at high risk of life-threatening aortic dissections. The condition is caused by mutations in the gene encoding fibrillin-1, an essential component in the formation of elastic fibers. While experimental findings in animal models of the disease have shown the involvement of transforming growth factor- $\beta$ (TGF- $\beta$ )- and angiotensin II-dependent pathways, alterations in the vascular extracellular matrix (ECM) may also play a role in the onset and progression of the aortic disease. Lysyl oxidases (LOX) are extracellular enzymes, which initiates the formation of covalent cross-linking of collagens and elastin, thereby contributing to the maturation of the ECM. Here we have explored the role of LOX in the formation of aortic aneurysms in MFS. We show that aortic tissue from MFS patients and MFS mouse model $\left(F b n 1^{\mathrm{C} 1039 \mathrm{G} /+}\right)$ displayed enhanced expression of the members of the LOX family, LOX and LOX-like 1 (LOXL1), and this is associated with the formation of mature collagen fibers. Administration of a LOX inhibitor for 8 weeks blocked collagen accumulation and aggravated elastic fiber impairment, and these effects correlated with the induction of a strong and rapidly progressing aortic dilatation, and with premature death in the more severe MFS mouse model, Fbn $1^{\mathrm{mgR} / \mathrm{mgR}}$, without any significant effect on wild type animals. This detrimental effect occurred preferentially in the ascending portion of the aorta, with little or no involvement of the aortic root, and was associated to an overactivation of both canonical and non-canonical TGF- $\beta$ signaling pathways. The blockade of angiotensin II type I receptor with losartan restored TGF- $\beta$ signaling activation, normalized elastic fiber impairment and prevented the aortic dilatation induced by LOX inhibition in Fbn $1^{\mathrm{C} 1039 \mathrm{G} /+}$ mice. Our data indicate that LOX enzymes and LOX-mediated collagen accumulation play a critical protective role in aneurysm formation in MFS.
\end{abstract}

(c) 2015 Elsevier Ltd. All rights reserved.

\section{Introduction}

Marfan syndrome (MFS) is an autosomal dominant connective tissue disorder caused by mutations in the gene encoding fibrillin-1 (FBN1), an essential component in the formation of elastic fibers [1,2]. The disorder has pleiotropic manifestations in the skeletal (overgrowth),

\footnotetext{
* Corresponding author at: Centro de Biología Molecular "Severo Ochoa”, Consejo Superior de Investigaciones Científicas (C.S.I.C.)/Universidad Autónoma de Madrid (U.A.M.), Nicolás Cabrera 1, E-28049 Madrid, Spain. Tel.: + 3491196 4505; fax: + 3491 1964420.

E-mail address: frodriguez@cbm.csic.es (F. Rodriguez-Pascual).

1 O. Busnadiego, D. Gorbenko del Blanco and J. González-Santamaría contributed equally as co-first authors.

${ }^{2}$ G. Egea and F. Rodriguez-Pascual contributed equally as co-corresponding authors.
}

ocular (lens luxation) and cardiovascular (progressive proximal aorta dilatation and mitral valve prolapse) systems. Prognosis of MFS is dominated by the cardiovascular phenotype, with a high risk of life-threatening aortic dissection.

Although the pathogenesis of the aortic disease could be explained by simple tissue alteration imposed by defective assembly of elastin into elastic fibers, recent investigations have shown a more complex scenario, with involvement of transforming growth factor- $\beta$ (TGF- $\beta$ ) and angiotensin II-dependent pathways, as well as matrix-dependent alterations [3-6]. To further complicate matters, the aortic phenotype of MFS patients vary widely, with small aneurysms that rupture very soon whereas other larger dilatations remain asymptomatic, even among family members harboring the same FBN1 mutation [7]. These observations suggest that epistatic interactions with other factors 
distinct to FBN1 may be important, and the identification of gene candidates for such modifiers is of clinical interest. While the search for such genes has been focused on those co-expressed with FBN1 in mouse aorta, without a clear definition of those being directly implicated in the progression of aortic dilatations, genes involved in the process of aortic remodeling, especially those belonging to the ECM or ECMremodeling categories constitute highly attractive candidates [8]. Particularly interesting is the case of the lysyl oxidase (LOX) family of matrix remodeling enzymes. These enzymes, for which five members have been described in mammals (the canonical LOX, and four LOX-like from 1 to 4 ), catalyze the conversion of lysine and hydroxylysine groups of collagen and elastin into highly reactive aldehydes, which eventually condense with other aldehyde group or intact lysine residue to form a variety of inter- and intrachain cross-linkages [9]. LOX family members are essential factors for the maturation of the ECM as evidenced in mouse models deficient in LOX and LOXL1, and in experimental models under pharmacological inhibition of LOX enzymatic activity, which have been shown to display defective ECM [10-13]. Interestingly, both genetic and pharmacological inactivation of LOX enzymatic activity is linked to cardiovascular malformations, evidences that support a fundamental role of LOX family members in vascular ECM homeostasis.

In the context of MFS, conflicting reports exist on the contribution of LOX to aortic pathology. Early work reported that elastin cross-linking was reduced in the aorta of MFS patients as compared to healthy subjects [14]. However, other studies did not find any significant alteration in LOX activity or LOX mRNA in skin fibroblasts or vascular smooth muscle cells isolated and cultured from MFS patients [15,16]. More recently, defects in collagen microarchitecture (higher collagen content and increased collagen cross-linking), together with increased tissue stiffness have been reported in the aneurysms of MFS mice [17]. Therefore, the question of whether LOX enzymes are involved in aortic disease progression of MFS still remains unclear. To address this issue, we have characterized the expression of LOX isoforms in aortic tissue of MFS patients and mice, and analyzed their impact on aneurysm formation in animal models of the disease.

\section{Results}

2.1. LOX and LOXL1 expression is upregulated in aortic tissue from MFS patients and in a MFS mouse model

Surgical specimens from the ascending aorta of 16 patients (clinical characteristics in Supp. Table 1) were analyzed by immunohistochemistry for the expression of LOX isoforms (LOX and LOX-like 1 to 4) as well as for elastic fiber and collagen architecture visualized by Verhoeff-Van Gieson's (VVG) and Picrosirius red (PSR) staining, respectively. Normal thoracic aortas were retrieved from 8 organ transplant donors. As shown in Fig. 1, aneurysmal tissue from MFS patients displayed disorganized elastic network and collagen fiber accumulation and disorder as compared with healthy aorta. Interestingly, collagen visualization by PSR under polarized light showed evident positive birefringence in MFS medial aorta; in marked contrast to normal aorta, which displayed an almost black background. While the presence of positively birefringent collagen scaffolds does not directly demonstrate enhanced cross-linking, these observations indicate the potential involvement of LOX upregulation in the formation of mature collagen
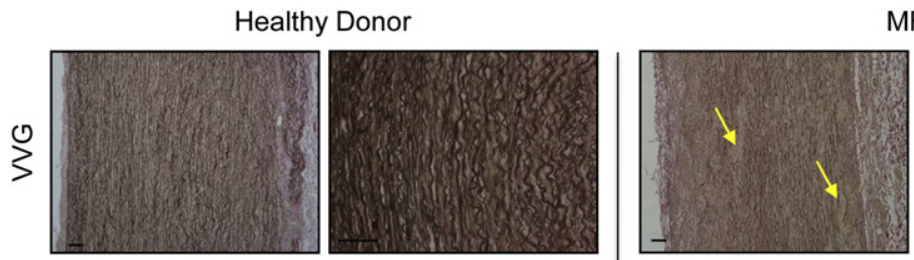

MFS
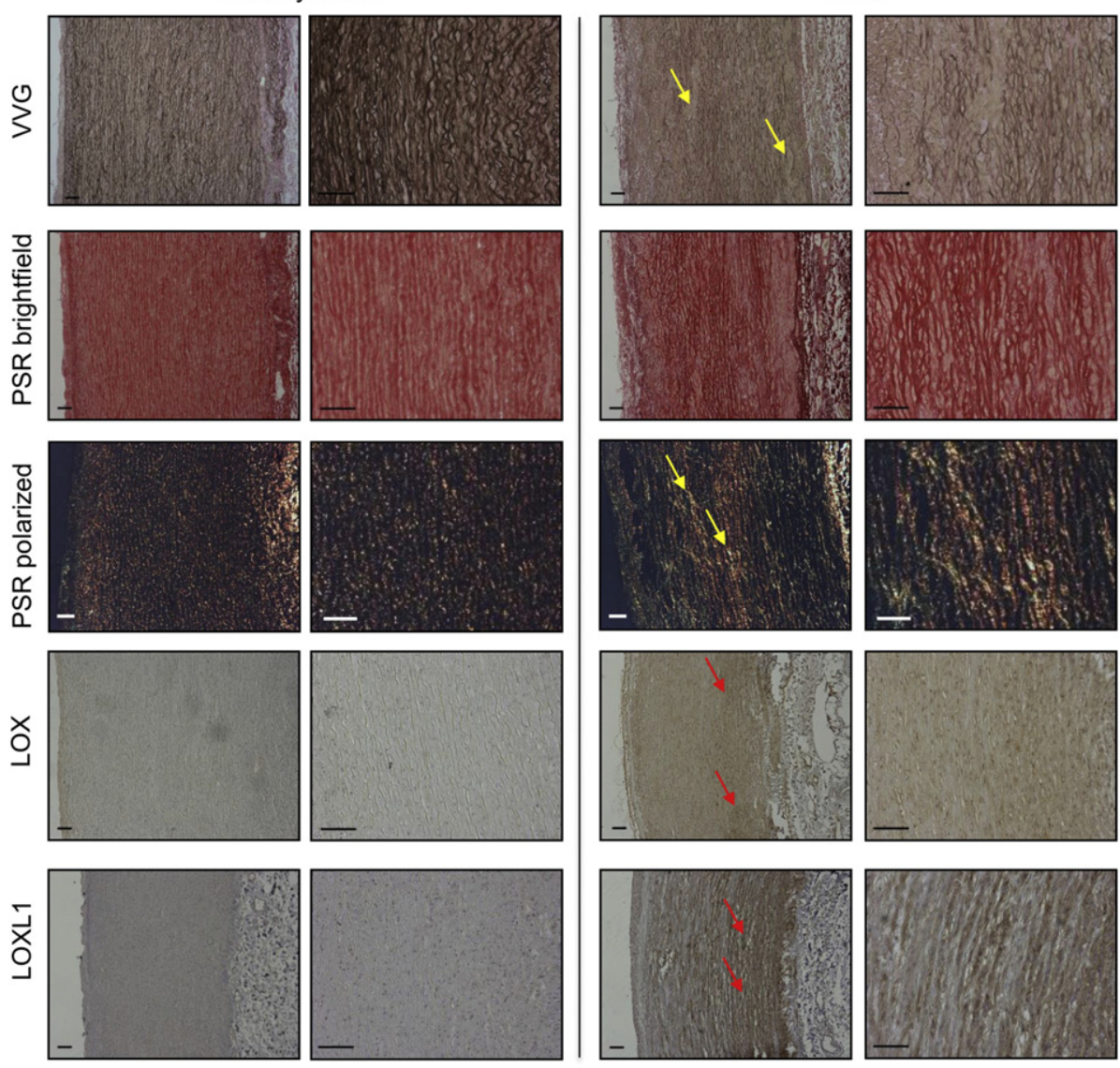

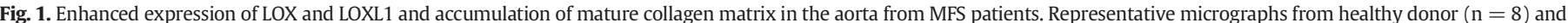

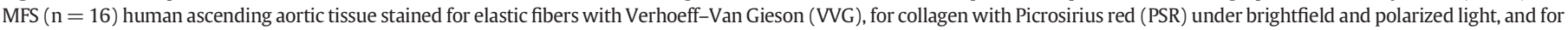

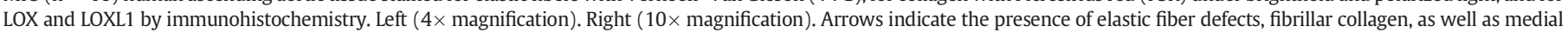
areas showing immunostaining for LOX and LOXL1. Scale bar: $0.5 \mathrm{~mm}$. 
bundles in the aorta of MFS patients. Immunohistochemical analysis showed enhanced expression of LOX and LOXL1 in the aortic medial layer of MFS patients compared to normal aorta (Fig. 1). We did not find differential expression of the remaining isoforms of LOXL (LOXL24 ) in MFS versus normal aorta (data not shown). We confirmed the upregulation of LOX isoform by immunoblotting using protein extracts isolated from healthy and MFS aorta (Supp. Fig. 1A). Available antibodies for the LOXL1 did not work for immunoblotting in our hands (data not shown). Aorta tissues were also used for determination of LOX activity by $\mathrm{H}_{2} \mathrm{O}_{2}$ production with Amplex Red. While specific activity could not be detected in extracts isolated from healthy aorta, samples from MFS patients showed measurable levels of enzymatic activity (Supp. Fig. 1B). Additionally, medial areas of MFS and control aorta sections were laser-capture microdissected and RNA extracted from these tissue portions. As shown in Supp. Fig. 1C, MFS samples showed enhanced mRNA expression of LOX and LOXL1 compared with controls, in agreement with the results obtained by immunohistochemistry.

The expression of LOX isoforms and their association with alterations in elastic fiber and collagen network were also studied in a MFS mouse model. As previously reported, proximal ascending aorta of mice heterozygous for a missense mutation in Fbn1 gene $\left(\right.$ Fbn $\left.1^{\mathrm{C} 1039 \mathrm{G} /+}\right)$, a validated animal model of MFS [18], displayed significant elastic fiber fragmentation (Fig. 2A). Compared to wild type (WT), MFS mice showed evident collagen deposition, which, in analogy with MFS patients, displayed increased content of mature collagen, as visualized by PSR staining under polarized light. The ECM structural alterations observed in MFS mice occurred with upregulation of LOX protein, as assessed by immunohistochemistry and immunoblotting (Fig. 2A and B). These analyses are only shown for LOX, as the available antibody for LOXL1 did not function in mouse aortic tissue samples. Additionally, MFS mice aorta showed enhanced mRNA expression of Lox and Loxl compared with controls (Fig. 2C). These findings indicate that the expression of LOX and
LOXL1 isoforms is increased in aneurysmal tissues from MFS patients and in a mouse model of the disease, in association with enhanced deposition of mature collagen in the tunica media.

\subsection{Inhibition of LOX activity induces the dilatation of the ascending aorta in MFS mouse models}

In order to test whether the increased expression of LOX isoforms in the diseased aorta plays a significant role in aneurysmal formation, Fbn $1^{\mathrm{C} 1039 \mathrm{G} /+}$ mice were treated with $\beta$-aminopropionitrile (BAPN), a compound shown to irreversibly inhibit LOX activity in vitro and in vivo [19]. As shown in Fig. 3A and B, inhibition of LOX enzymatic activity significantly attenuated the formation of mature birefringent collagen fibers in the medial layer of MFS mice as assessed by PSR under polarized light. Consistently, evaluation of LOX enzymatic activity by Amplex Red showed that MFS mice displayed higher values compared to wild type animals, and that they were significantly reduced by BAPN administration (Fig. 3C). We have also determined the levels of collagen cross-links (HPLC) and total collagen (colorimetric hydroxyproline assay). As shown in Fig. 3D, MFS mice displayed a modest though significant increase in hydroxylysylpyridinoline (HP) levels as compared to wild type. LOX inhibition with BAPN normalized the amount of cross-links to control levels. Levels of lysyl pyridinoline cross-links, which are usually about ten times lower than HP in most tissues, were below detection level in mouse samples under our experimental conditions (data not shown). Biochemical determination of total collagen revealed a higher content in MFS compared to wild type mice, and a significant reduction in BAPN-treated MFS mice, an observation indicating that LOX-mediated activity is required for collagen deposition in aorta (Fig. 3E). We then studied the progression of aortic aneurysm by echocardiogram after 4 and 8 weeks of BAPN treatment (Fig. 4A and B). LOX inhibition induced a strong and rapidly progressing
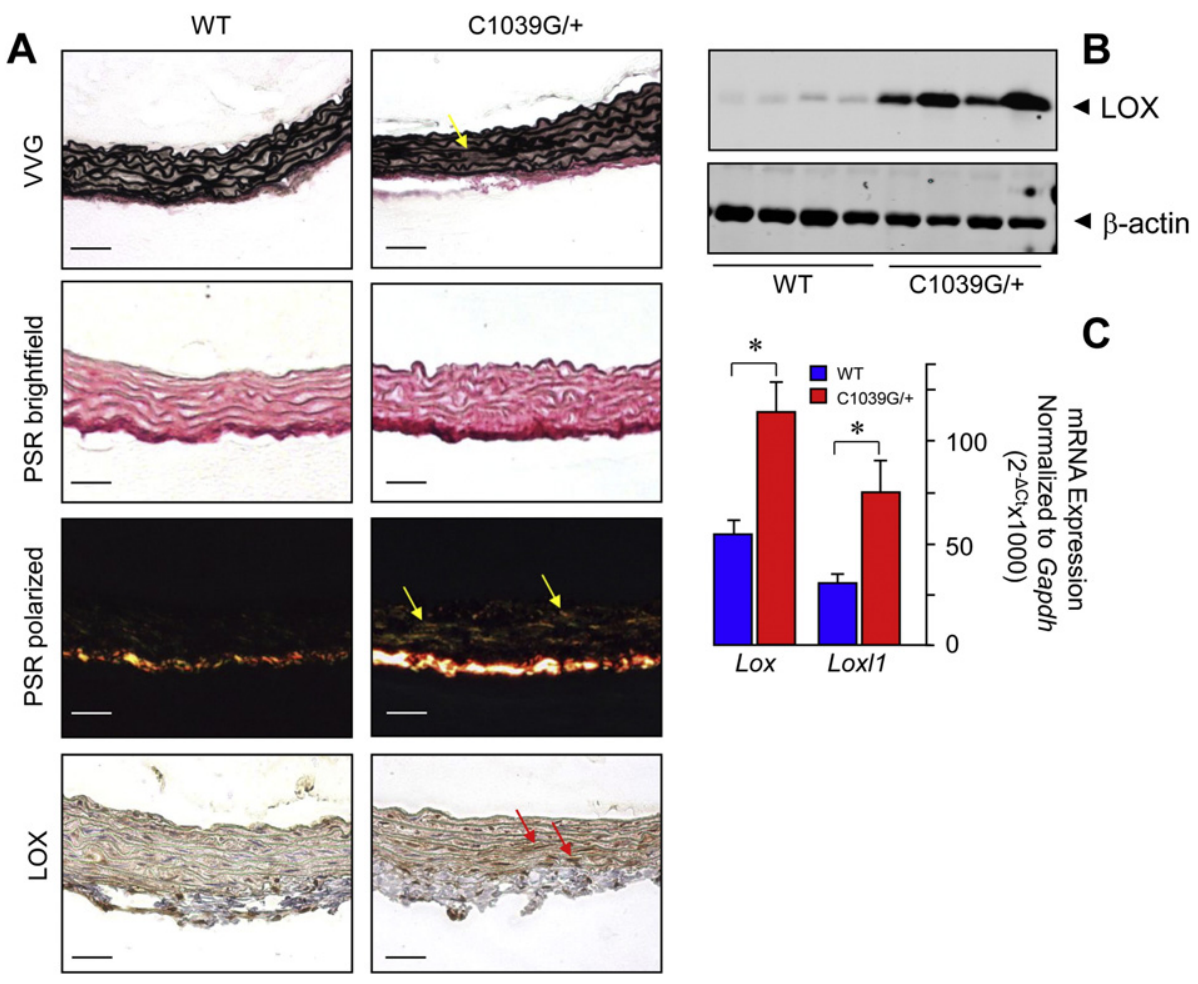

C

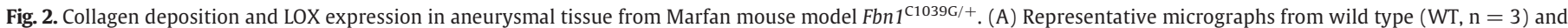

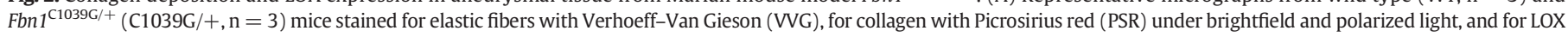

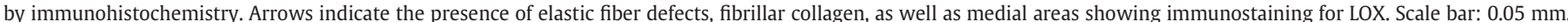

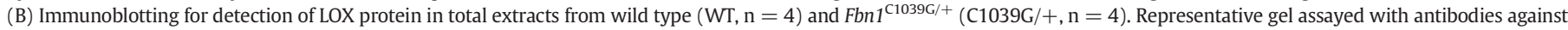

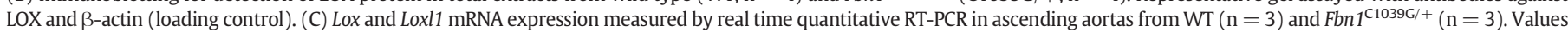
are means + SEM. ${ }^{*} \mathrm{P}<0.05$. 
A

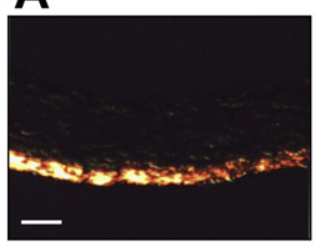

WT

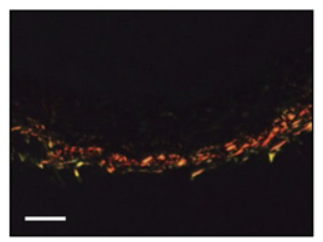

WT+BAPN

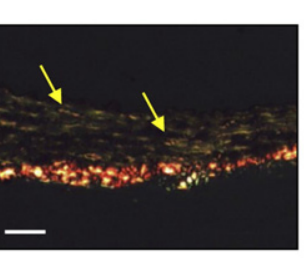

C1039G/+

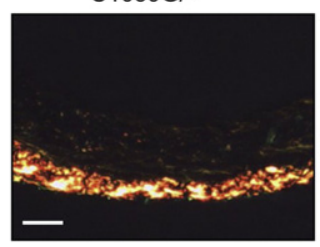

C1039G/++ BAPN
B

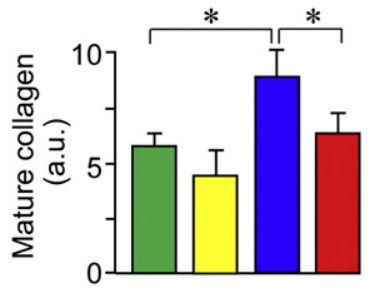

D

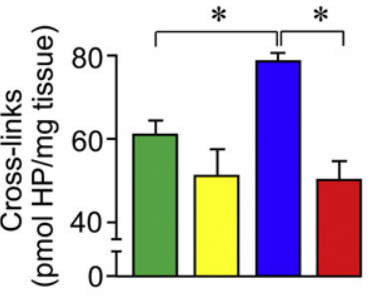

$\begin{array}{ll}\square W T & \square \mathrm{C} 1039 \mathrm{G} /+ \\ \square W T+B A P N & \square \mathrm{C} 1039 \mathrm{G} /++\mathrm{BAPN}\end{array}$

$\begin{array}{ll}\square W T & \square \mathrm{C} 1039 \mathrm{G} /+ \\ \square W T+B A P N & \square \mathrm{C} 1039 \mathrm{G} /++\mathrm{BAPN}\end{array}$

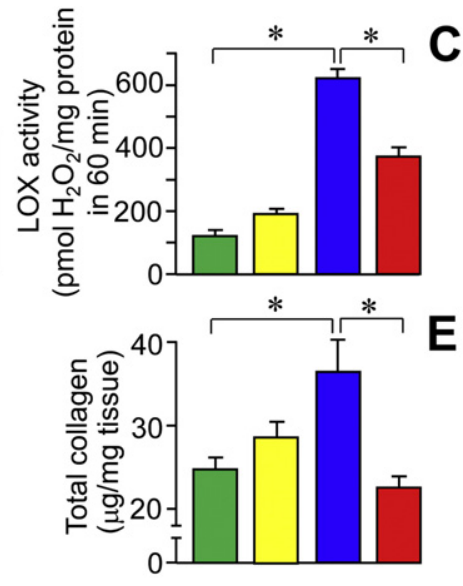

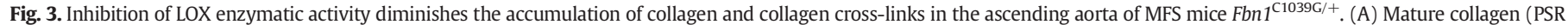

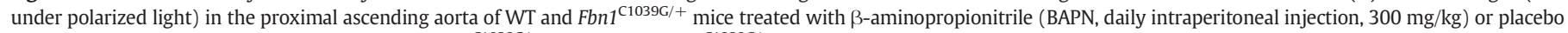

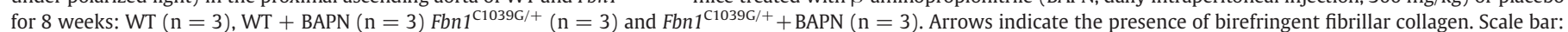

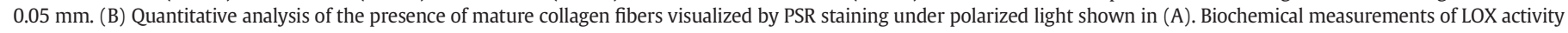

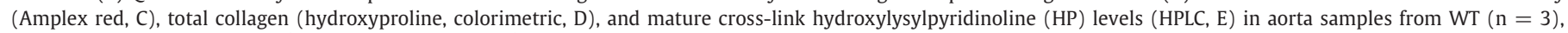

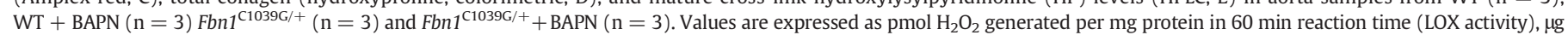
(total collagen) and pmol (crosslinks, HP) per mg wet tissue. Values are mean $\pm \mathrm{SEM}$. ${ }^{*} \mathrm{p}<0.05$.

dilatation of the ascending portion of the aorta of $F b n 1^{\mathrm{C1039G} /+}$ mice compared to baseline values, without any significant effect on WT animals. Interestingly, BAPN treatment impacted the ascending aorta diameter in this mouse models in the absence of a significant effect on the aortic root, which progressively dilated in MFS mice, but whose rate was not modified by LOX inhibition. Morphometric analyses of aorta at the end of the 8-week in vivo trial confirmed the echocardiographic observations (Fig. 4C). We have then investigated whether

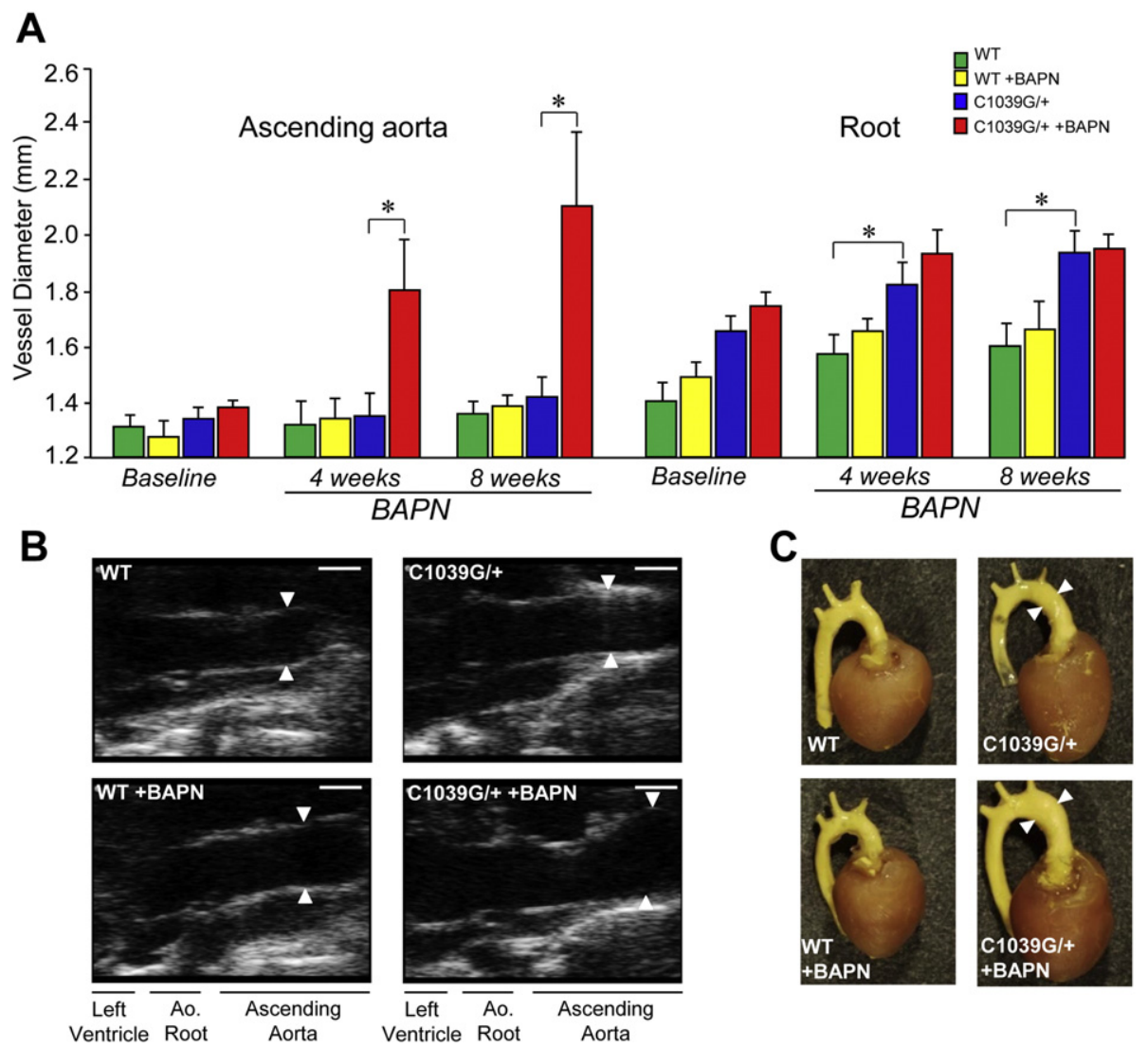

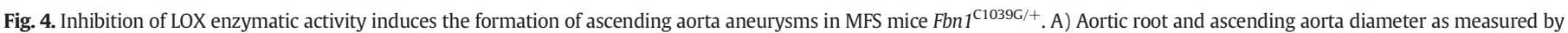

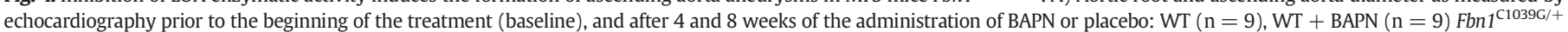

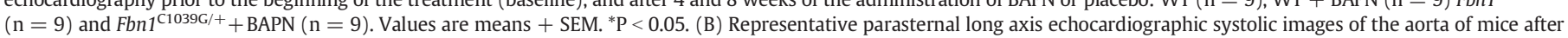
8 weeks of treatment. Arrows indicate the dimension of the ascending aorta. Scale bars: $1 \mathrm{~mm}$. (C) Gross morphology of representative aortas from mice after 8 weeks of treatment. 
different histological and signaling processes are taking place in the aortic root versus the ascending aorta that might explain the response of these aorta portions to LOX inhibition. Histological analysis of the elastic fiber network shows that BAPN administration affected mainly the ascending aorta, with less involvement of the root (Fig. 5A and B). TGF$\beta$-dependent signaling pathways have been invoked to be implicated in the pathogenesis of the MFS aortic disease [3-6]. Therefore, we have investigated whether LOX inhibition altered the extent of activation of both canonical (Smad-dependent) and non-canonical (ERK-dependent) TGF- $\beta$ signaling pathways. As shown also in Fig. $5 \mathbf{C}$ and $\mathbf{D}$, BAPN administration induced a significant increase in both phosphoSmad2 and phospho-ERK1/2 in the ascending aorta, but not in the root. We have also analyzed whether BAPN administration altered the expression of collagen genes (Col1a1 and Col3a1), as well as matrix metalloproteases (Mmp2 and Mmp9) and its inhibitor Timp4, some of them reported to be abnormally expressed in aneurysmal tissues [20]. Except for Col1a1, which was significantly upregulated by BAPN in MFS mice, but not in wild type, the expression of the remaining genes remained essentially unchanged (Supp. Fig. 2). We have also analyzed the effect of LOX inhibition in homozygous mgR mice, a more aggressive MFS model with hypomorphic expression of FBN1 protein [21]. As shown in Fig. 6A-C, mgR mice also reproduced the aortic phenotype induced by inhibition of LOX activity: a progressive growth of ascending aorta as observed after 4 and 8 weeks of BAPN administration. Similarly to $F b n 1^{\mathrm{C} 1039 \mathrm{G} /+}$ mice, BAPN did not significantly modified the rate of dilatation of aortic root in the mgR model (Supp. Fig. 4). In consistence with these results, LOX inhibitor sharply shortened the survival of homozygous mgR mice as compared with animals treated with vehicle (Fig. 6D). Post-mortem macro- and microscopic examination of $\mathrm{mgR}$ mice under placebo and BAPN treatment has revealed that BAPN accelerated the deterioration of the aorta, and, interestingly, precipitated the development of the cardiomyopathy, a typical manifestation of this model of the disease (Supp. Fig. 3) [22].
2.3. The angiotensin II receptor blocker, losartan prevents aortic dilatation induced by LOX inhibition in MFS mice

The blockade of angiotensin II type I receptor (AT1R) has been shown to reduce aortic dilatation in MFS mice, and this observation is the basis of the clinical use of this drug to halt or slow down the progression of aneurysmal disease in MFS patients [23-25]. We studied the effect of losartan on BAPN-mediated aortic disease exacerbation in Fbn1 ${ }^{\mathrm{C} 1039 \mathrm{G} /+}$ mice. As shown in Fig. 7A, losartan treatment prevented the ascending aorta dilatation induced by LOX inhibition in MFS mice. Histological examination by VVG staining revealed that losartan administration normalized elastic fiber fragmentation in vehicle or BAPNtreated MFS mice (Fig. 7B and C). We have also analyzed the extent of collagen maturation in animals treated with BAPN and losartan. As shown in Fig. 7B and D, treatment with losartan on top of BAPN did not modify the collagen matrix, which appears essentially the same as in the BAPN only group or in wild type animals. The extent of the activation of both canonical Smad2 and non-canonical ERK TGF- $\beta$-dependent signaling pathways was studied by immunohistochemistry in MFS mice treated with BAPN and losartan. As shown in Fig. 7E, losartan administration restored the basal levels of activation of both signaling pathways.

\section{Discussion}

The most serious complications associated with MFS involve the development of life-threatening thoracic aortic aneurysms. While experimental findings in animal models of the disease have indicated the involvement of TGF- $\beta$ - and angiotensin II-dependent pathways, as well as the contribution of matrix-dependent effects or augmented mechanical stress, a complete picture about the pathogenetic mechanism driving the onset and progression of the aortic disease has not been achieved. In the context of the mechanobiology, the aortic ECM has
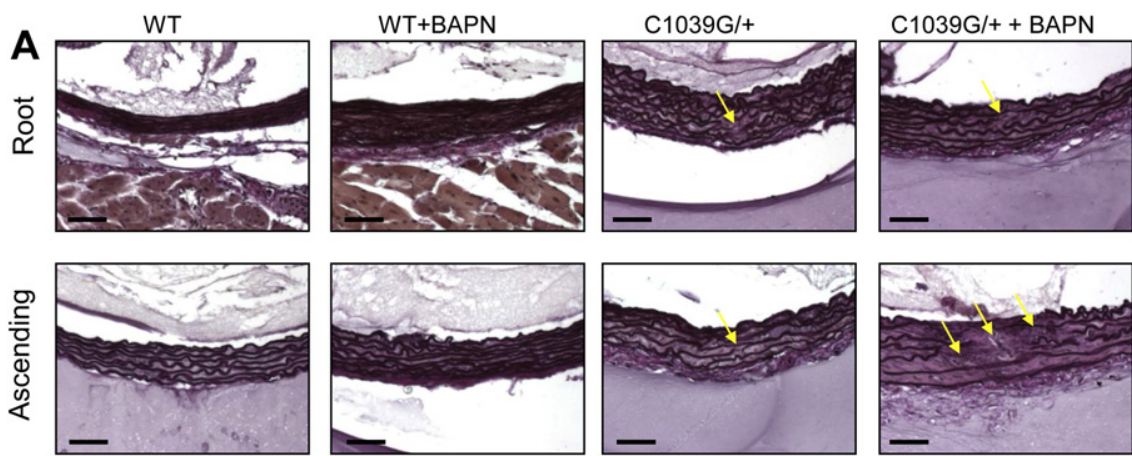

C

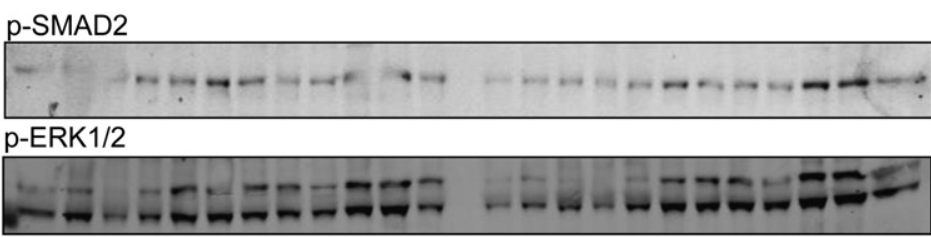

$\beta$-actin
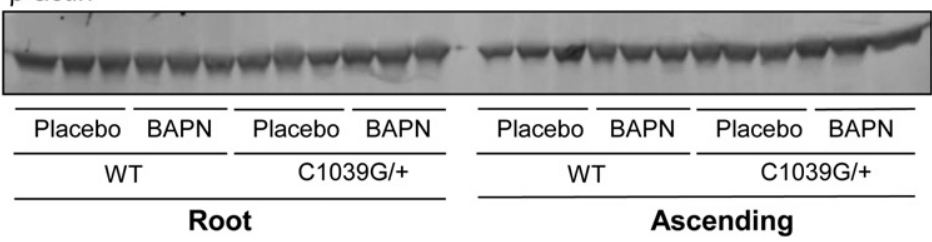
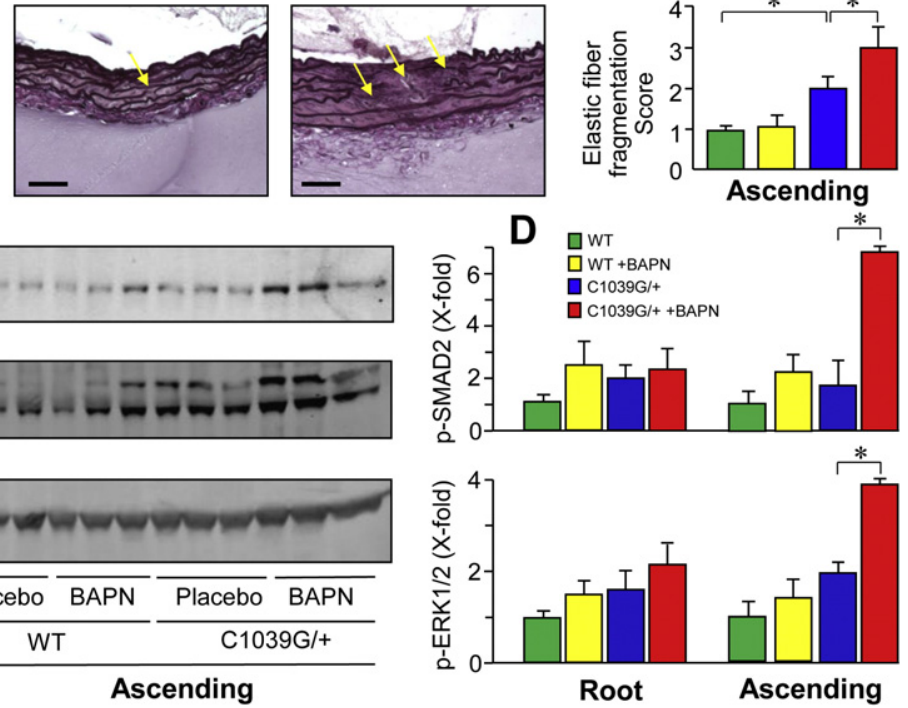

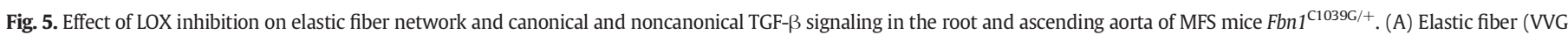

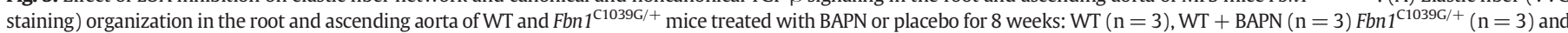

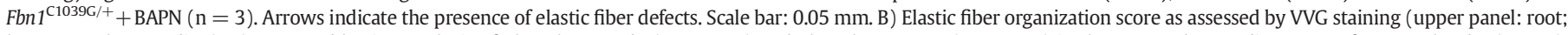

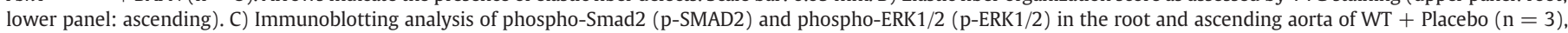
$\mathrm{WT}+$ BAPN $(\mathrm{n}=3) \mathrm{Fbn}^{\mathrm{C} 1039 \mathrm{G} /+}+$ Placebo $(\mathrm{n}=3)$ and Fbn $1^{\mathrm{C} 1039 \mathrm{G} /+}+\mathrm{BAPN}(\mathrm{n}=3)$ mice. (C) Protein band intensities normalized to $\beta$-actin. Values are means + SEM. ${ }^{*} \mathrm{P}<0.05$. 

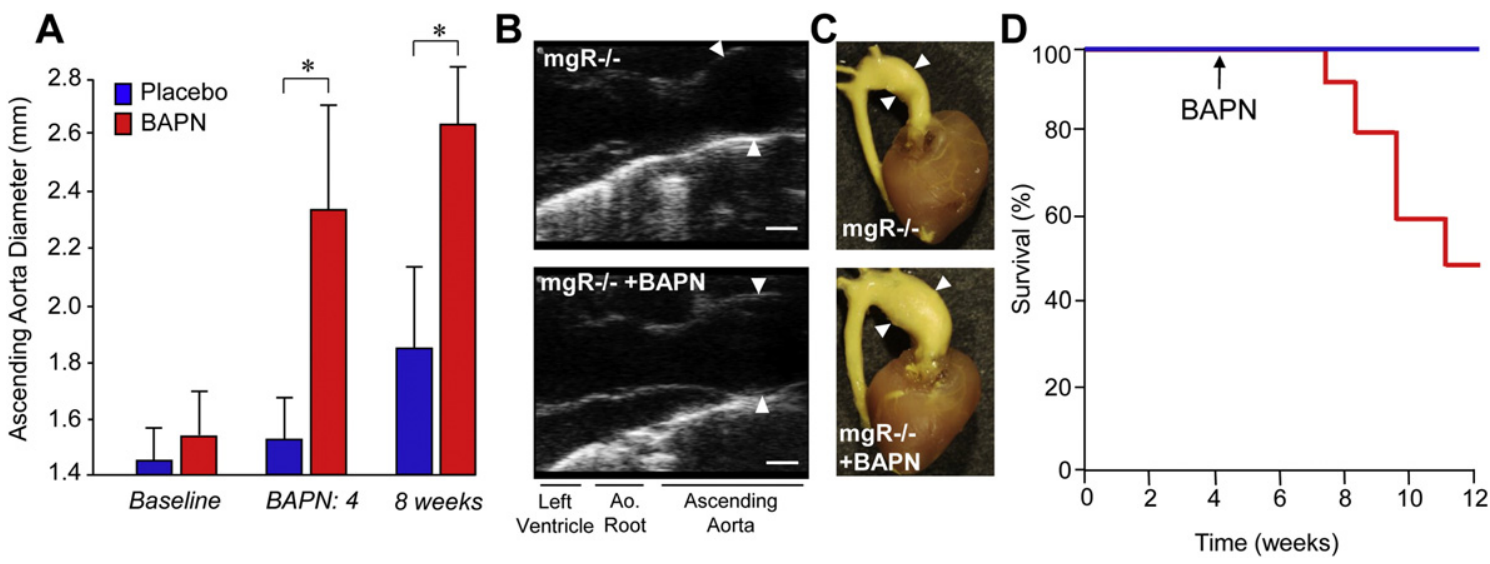

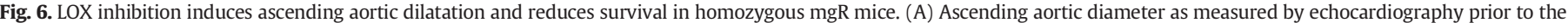

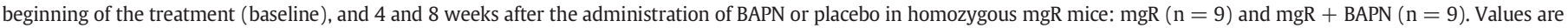

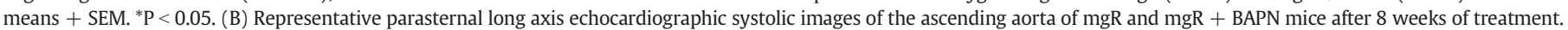

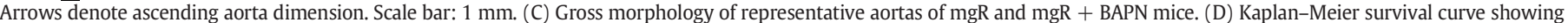
diminished life span of $\mathrm{mgR}+\mathrm{BAPN}$ as compared with $\mathrm{mgR}$ group.

been shown to play a fundamental role in sensing and responding to mechanical loading $[26,27]$. Main components of vessel wall ECM include elastin and collagen, which are responsible for the properties of recoil and strength, respectively, typical of a healthy aorta [28]. Both collagen and elastin fibers must be cross-linked in order to exhibit these properties, observations that support the notion that LOX family members are essential factors for the maturation of the ECM [29].

Here we report that the aorta of MFS patients and mouse models displays increased expression of LOX and LOXL1 isoforms. These results are in agreement with a previous evidence of elevated levels of nonreducible lysyl oxidase-initiated collagen cross-links (hydroxylysyl pyridinoline/lysyl pyridinoline cross-links) in the aorta from MFS patients, though the increase in the expression of LOX isoforms had not been previously reported [17]. Increased expression levels of LOX correlate with enhanced deposition of mature collagen fibers within the media layer. In fact, together with the presence of severe elastic lamellar disruption, a common histopathological pattern consistently found in MFS is the accumulation of collagenous material, and a concomitant increase in tissue stiffness [30-33]. To this respect, we have recently reported that vascular smooth muscle cells isolated from the aorta of MFS patients display increased cell and matrix stiffness as a result of increased expression of contractile proteins and collagen [34]. While it is usually assumed that collagen deposition and stiffness are features of the disease, it is still unknown whether they are a consequence of chronic damage, being the substrate for the dilatation and further rupture, or constitute an active response opposing pathological remodeling. This has in fact important clinical implications as the measurement of aortic stiffness has been largely used for diagnosis and prognosis in affected individuals, though some recent clinical studies claimed that increased aortic stiffness is associated with slower rate of aneurysm progression rather than with augmented dilatation and rupture risk $[32,33]$. Our data may shed light on this controversy as we found that the disruption of the accumulation of fibrillar collagen by inhibition of LOX activity induces the development of aortic aneurysms in MFS mice. In consistence with these results, a case report described a spontaneous arterial wall dissection associated with ECM disorganization and dramatically reduced LOX expression [35]. Therefore, our results indeed indicate that collagen deposition and cross-linking constitute a beneficial response against tissue deformation, with LOX enzymes playing a critical role in this action.

In our study, inactivation of LOX activity is achieved by the use of the broad inhibitor, BAPN [19]. Interestingly, at the dosage (300 mg/kg) and time (a maximum of 8 weeks) regime used in our study, we did not observe any particular effect on the aorta of wild type animals, suggesting that MFS mice are somehow predisposed to the formation of ascending aorta lesions. Noteworthy, recent evidences have shown that infusion of BAPN induces aortic aneurysm and rupture in mice only in conjunction of hypertension, either by administration of angiotensin II or deoxycorticosterone acetate (DOCA)-salt $[10,13]$. These observations indicate the requirement of an alteration in the vascular ECM together with an increase in the hemodynamical loading as a necessary combination for the development of the aortic dilatation. We propose that the factor predisposing the aorta of MFS mice to develop ascending aorta dilatations under LOX inhibition is the existence of increased mechanical stress, presumably due to an elastically impaired ECM. Interestingly, and very consistent with the echocardiographic and histological analyses, this effect was more evident in the ascending portion of the aorta than in the root. Although other explanations are feasible, such as the different embryonic origin for smooth muscle cells populating these aorta portions [36], the fact that LOX inhibition preferably affects the ascending aorta further supports the notion that mechanical stress is a fundamental driver of the disease, as it receives the highest impact from the blood pressure. In fact, this observation is highly reminiscent of the aortic pathology usually found in bicuspid aortic valve (BAV) patients, where hemodynamic derangements have been proposed to cause alterations in the ascending aorta, and suggests that collagen and collagen crosslinking play an important role in providing this segment of the aorta with the mechanical properties required to withstand with the hemodynamic load [37]. Taken together, our model may be conceived as an association of MFS and BAV, a human condition that has been reported to result in a more severe aortic involvement, with a higher percentage of patients requiring surgical intervention [38].

Considering the matrix-supporting role of LOX enzymes, the deleterious effect of LOX inhibition on aneurysmal progression might directly be explained by deficient matrix cross-linking and subsequent impairment of tissue integrity. We have nevertheless explored whether LOX inhibition may alter signaling pathways directly involved in the development of thoracic aortic aneurysms, in particular TGF- $\beta$-dependent pathways [3-6]. Here we show that LOX inhibition results in an overactivation of both canonical (Smad) and non-canonical (ERK) TGF- $\beta$-signaling pathways, indicating that LOX or LOX-remodeled matrix is an exquisite regulator of TGF- $\beta$ signaling activation. We also show that AT1R antagonism with losartan prevents BAPN-mediated aorta deterioration, while restoring basal levels of activation of TGF- $\beta$ dependent pathways. The fact that losartan show effectiveness in preventing aortic disease in MFS mice with or without inhibition of LOX activity positions the AT1R as a central receptor in the pathogenetic route leading to aortic aneurysms. Although still a matter of controversy, current hypotheses on aortic aneurysm pathogenesis in MFS invoke mechanosignaling-dependent pathways, such as angiotensin II/AT1R 

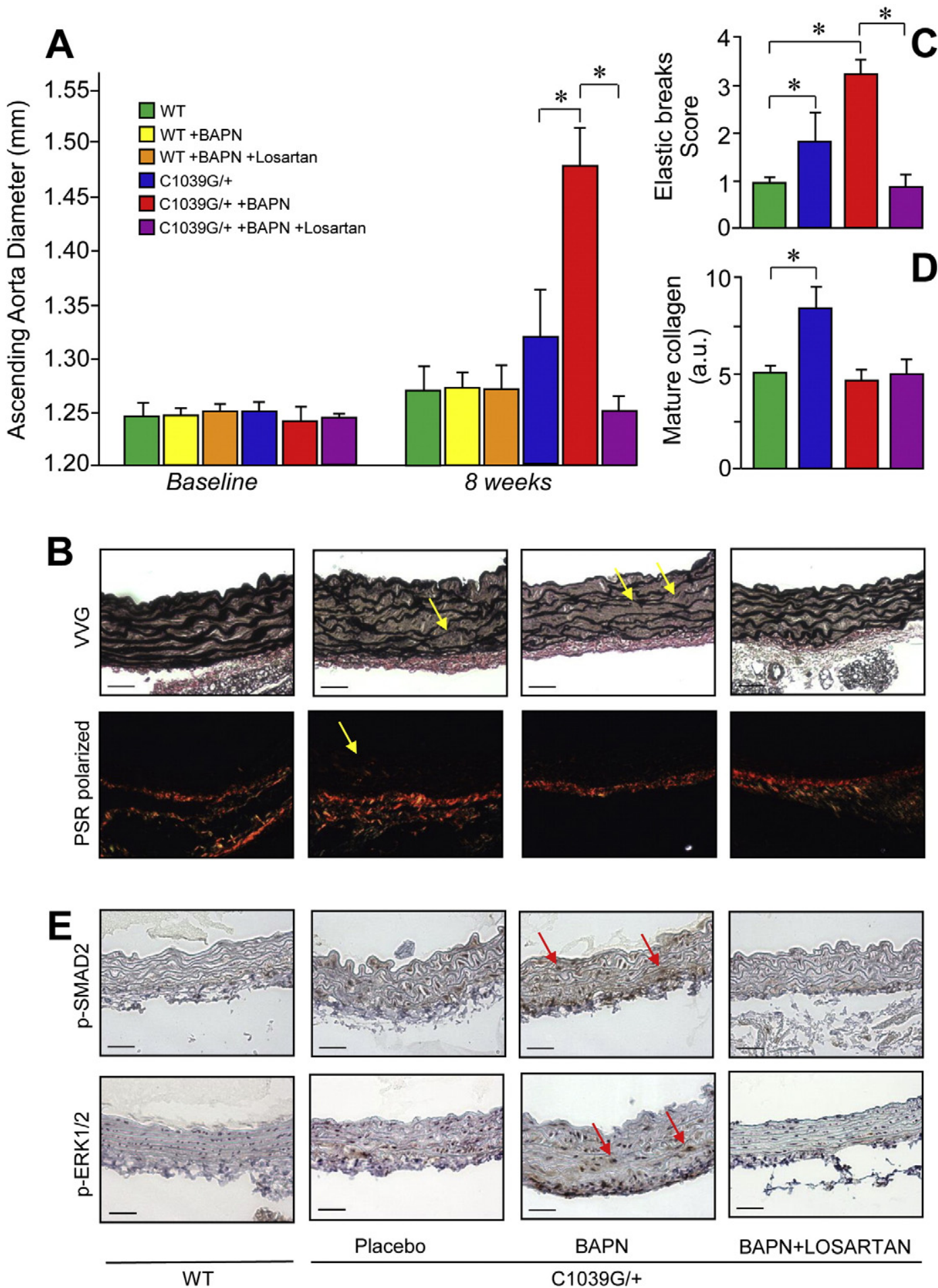

BAPN+LOSARTAN

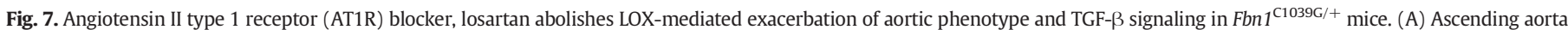

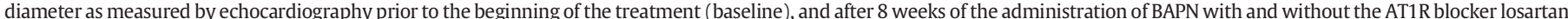

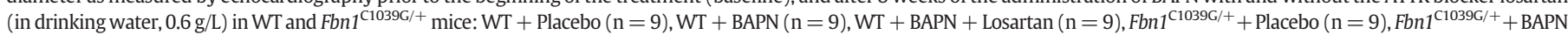

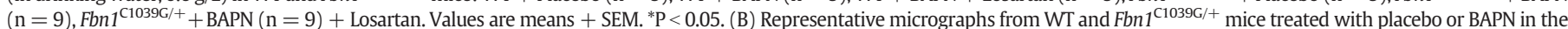

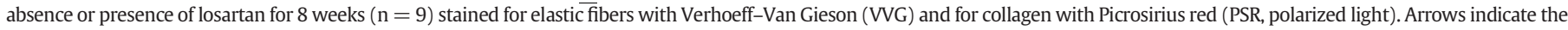

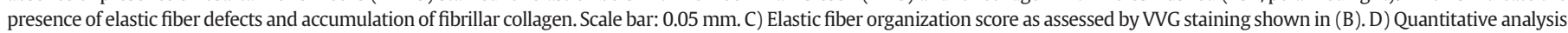

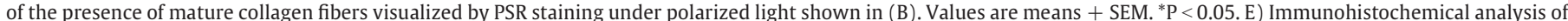

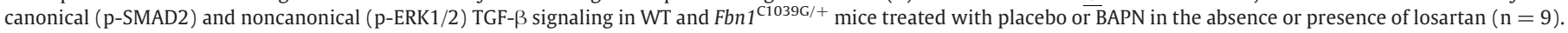
Arrows indicate the presence of medial areas showing immunostaining for p-SMAD2 and p-ERK1/2. Scale bar: $0.05 \mathrm{~mm}$.

coupled to downstream TGF- $\beta$ signaling, as a major driver of disease progression [6,26,27,39]. Based on this model, LOX inhibition may result in aggravation of the aortic pathology by two independent mechanisms.
On one hand, LOX inhibition might cause worsening of the aortic phenotype by weakening of the ECM and therefore augmentation of the hemodynamic stress and subsequent TGF- $\beta$ signaling and 
TGF- $\beta$-dependent aortic responses. Properly cross-linked collagen might therefore play an entirely unanticipated protective role by limiting signaling events leading to the pathological remodeling. An alternative explanation is based on recent in vitro and in vivo evidences suggesting a direct role for LOX in the control of active TGF- $\beta$ through a regulatory TGF- $\beta /$ LOX feedback loop $[40,41]$. Considering this potential mechanism, reported to be essential for the regulation of matrix deposition during skeletal muscle development, LOX inhibition would rise to enhanced TGF- $\beta$ levels, and subsequently to a potentiation of its detrimental effects. In one way or another, by inhibition of the mechanosensor AT1R, losartan uncouples the driving force of the pathogenetic process, restoring basal levels of TGF- $\beta$ dependent pathways, and rendering the process not influenced by LOX inhibition.

In conclusion, our work supports a protective role for LOX enzymes in the development of aneurysms in MFS. Although more experimentation is required, based on our results, LOX and LOXL1 genes may be considered as a potential modifier of the phenotype of MFS, so that high levels of LOX/L expression might stabilize aneurysms whereas low levels may be associated to increased risk of aortic rupture. LOX isoforms are extracellularly secreted and can therefore be measured in serum samples as an index of tissue expression [42]. Therefore, LOX may be used as a prognostic biomarker in MFS and this aspect is currently being investigated.

\section{Materials and methods}

\subsection{Patients}

Aortic tissue was obtained at the time of surgery from patients with Marfan syndrome undergoing repair of an aortic aneurysm or dissection in the Marfan Unit of Hospital Universitario "12 de Octubre", Madrid (Spain). All patients met established diagnostic criteria based on the revised Ghent nosology [43]. Normal thoracic aortas from organ donors for heart transplantation were obtained from the same hospital (see Supp. Table 1 for clinical data). Segments of aortic tissue (approximately $2 \times 2 \mathrm{~cm}$ ) were excised at surgery and either fixed in $10 \%$ buffered formalin. Removed samples were embedded in paraffin wax and processed for histological, immunohistochemical and gene expression studies. The clinical study was approved by the Ethics Committee at the Hospital Universitario 12 de Octubre, and patients were included after providing informed, written, and signed consent.

\subsection{Mice}

All mice were cared for under strict compliance with the Animal Care and Use Committee of the Johns Hopkins University School of Medicine. Mouse lines, Fbn $1^{\mathrm{C} 1039 \mathrm{G} /+}$ and mgR, with mutations in the Fbn1 gene, were used as validated MFS animal models [18,21]. Both lines were maintained on a C57BL/6 background, allowing for valid comparisons. In order to further accommodate the potential for temporal- or background-specific variation in genetic or pharmacological manipulation experiments, all comparisons were made between contemporary littermates. Animals were checked daily and all mice found dead were immediately necropsied to assess for evidence of aortic dissection. Mice were killed with an inhalation overdose of halothane (SigmaAldrich, St. Louis). Mice underwent immediate laparotomy, descending abdominal aortic transection, and Phosphate-Buffered Solution (PBS, pH 7.4) infusion through the left ventricle to flush out the blood. Mice for aortic histology had latex (Ward's Natural Science, Rochester) injected under low pressure into the left ventricular apex until it was visible in the descending abdominal aorta. Animals were then fixed for $24 \mathrm{~h}$ in $10 \%$ buffered formalin, after which the heart and aorta were stored in $70 \%$ ethanol. For immunoblotting analysis, LOX activity determination, and total collagen and collagen cross-links quantification, aorta samples were removed, flash frozen in liquid nitrogen and stored at $-80^{\circ} \mathrm{C}$. Samples for RNA isolation were stored in RNAlater (Sigma Aldrich) and stored at $-80^{\circ} \mathrm{C}$ until processing.

LOX inhibitor $\beta$-aminopropionitrile (BAPN, Sigma-Aldrich) was dissolved in PBS and administered daily by intraperitoneal injection, at a dose of $300 \mathrm{mg} / \mathrm{kg}$. Losartan was dissolved in drinking water and filtered to give a concentration of $0.6 \mathrm{~g} / \mathrm{L}$, giving an estimated daily dose of $40-60 \mathrm{mg} / \mathrm{kg} / \mathrm{day}$. Placebo groups received PBS (intraperitoneal) and drinking water, respectively. Treatment was initiated at 6 weeks of age and continued for 8 weeks.

\subsection{Echocardiography}

Nair hair removal cream was used on all mice the day prior to echocardiography. All echocardiograms were performed on awake, unsedated mice using a Vevo 660 V1.3.6 imaging system (Visualsonics, Toronto, Canada) and a $30 \mathrm{MHz}$ transducer. The aorta was imaged using a parasternal long axis view. Three separate measurements of the maximal internal systolic dimension at the sinus of Valsalva and proximal ascending aorta were made, and a mean was calculated. All imaging and measurements were performed by a cardiologist who was blinded to genotype and treatment arm. Mice were imaged at the beginning of the treatment ( 6 weeks of age, baseline), and then 4 and 8 weeks after BAPN and/or losartan treatment was initiated [25,44].

\subsection{Histological and immunohistochemical analysis}

The heart and thoracic aorta of the mice were dissected out en bloc. Transverse sections (2-3 mm long) of the entire thoracic aorta (root, ascending, transverse, and descending segments) were mounted in $4 \%$ agar prior to paraffin-embedding. Segments of human aortic tissues were directly embedded in paraffin wax.

Five-micron sections were stained with Verhoeff-van Gieson (VVG) for elastin. Slides were examined using an Eclipse T2000U microscope (Nikon Inc., Tokio, Japan). Four representative VVG images of each mouse aorta were assessed by 3 blinded observers and graded on a scale of 1 (indicating no elastic fiber breaks) to 4 (indicating extensive elastic fiber fragmentation) as previously described [25]. Tissue sections were also analyzed for collagen staining with Picrosirius Red (PSR) under brightfield and polarized light using Leica DM LS2 (Leica Microsystems, Solms, Germany). The presence of yellowish-orange birefringent staining under polarized light was taken as an indicative of mature collagen fibers [45].

For immunohistochemical analysis, cross-sectional slices of paraffinembedded aortic tissue were deparaffinized and counterstained with hematoxylin using standard protocols. Immunohistochemical stainings were performed to detect LOX, LOXL1, pSmad2 and pERK1/2. The following primary antibodies were used: anti-LOX (Ab31238, Abcam, Cambridge, Massachusetts), anti-LOXL1 (sc-166632, Santa Cruz Biotechnology, Dallas, Texas), anti-Phospho-Smad2 (3101, Cell Signaling Technology), and anti-Phospho-ERK1/2 (4695S, Cell Signaling Technology). Tissue samples were visualized using an Eclipse T2000U (Nikon Inc.). Quantification of stained sections was done by digital image analysis.

\section{5. $R$ NA and protein studies}

For RNA extraction, aortic tissue was homogenized in Qiazol (Qiagen, Venlo, The Netherlands) using an automatic bead homogenizer (FastPrep24, MP Biomedicals, Carlsbad, California). Medial layer of human aortic samples were laser microdissected using a PALM MicroBeam system (Zeiss, Jena, Germany). RNA was extracted using chloroform and precipitated with isopropanol. Transcript levels for the gene of interest were determined by real-time quantitative reverse transcription polymerase chain reaction (RT-PCR) using Taqman probes (Applied Biosystems, Life Technologies, Carlsbad, California) as previously described [46]. The following prevalidated probes were used to detect 
specific transcripts: Mm99999915_g1 (Gapdh), Mm00495386_m1 (Lox), Mm01145738_m1 (Loxl1), Mm01302039_g1 (Col1a1), Mm01254476_m1 (Col3a1), Mm00439498_m1 (Mmp2), Mm00442991_m1 (Mmp9), Mm01184417_m1 (Timp4), Hs03929097_g1 (GAPDH), Hs00942480_m1 (LOX), and Hs00935937_m1 (LOXL1). Aortic proteins were extracted and assayed by immunoblotting using specific primary antibodies and species-appropriate secondary antibodies for the LI-COR system (LI-COR Biosciences, Lincoln, Nebraska).

\subsection{LOX activity determination}

Aortic tissues were homogenized in $4 \mathrm{M}$ urea in $0.02 \mathrm{M}$ borate buffer ( $\mathrm{pH} 8.0$ ) at $4{ }^{\circ} \mathrm{C}$, and all homogenate was centrifuged at $15,000 \mathrm{~g}$ for $30 \mathrm{~min}$ at $4{ }^{\circ} \mathrm{C}$. The supernatants were analyzed immediately for LOX activity by measuring concomitant $\mathrm{H}_{2} \mathrm{O}_{2}$ production using Amplex red (Life Technologies) as a fluorogenic probe and cadaverine (SigmaAldrich) as LOX substrate, in the presence of horseradish peroxidase [47]. Fluorescence intensity was determined for test samples with and without $500 \mu \mathrm{M}$ BAPN and specific LOX activity calculated using $\mathrm{H}_{2} \mathrm{O}_{2}$ standard and expressed as pmol $\mathrm{H}_{2} \mathrm{O}_{2} / \mathrm{mg}$ protein in 60 min reaction time.

\subsection{Collagen and collagen cross-linking analysis}

Aorta tissue portions were hydrolyzed $\left(95^{\circ} \mathrm{C}, 20 \mathrm{~h}\right.$ ) in $6 \mathrm{M} \mathrm{HCl}$ and collagen (hydroxyproline) and mature trivalent cross-links, hydroxylysylpyridinoline (HP) and lysylpyridinoline (LP), analyzed by a colorimetric assay (Total Collagen Assay, Quickzyme, Leiden, The Netherlands) and by HPLC, respectively [48]. The amount of collagen and of the cross-link hydroxylysylpyridinoline (HP) is expressed as $\mu \mathrm{g}$ or pmol per mg wet tissue, respectively.

\subsection{Statistical analysis}

Data represent mean \pm SEM. Unpaired 2-tailed Student $t$ tests were used to determine the significance between two groups, assuming significance at $P<0.05$. Analyses between multiple groups used two-way ANOVA, with $P<0.05$ considered statistically significant. Statistical analysis was performed using GraphPad Prism software (La Jolla, California).

\section{Conflict of interest}

The authors declare they have no conflicts of interest.

\section{Disclosures}

None.

\section{Acknowledgements}

This work was supported by grants from Ministerio de Economía y Competitividad (Plan Nacional de I + D + I: SAF2012-34916), and Comunidad Autónoma de Madrid: 2010-BMD2321, FIBROTEAM Consortium, to F.R-P.; Howard Hughes Medical Institute, Smilow Center for Marfan Syndrome Research, The Marfan Foundation, and the National Institutes of Health (AR41135 and AR049698) to H.C.D.; and Ministerio de Economía y Competitividad (Plan Nacional de I + D + I: BFU201233932), Fundación Ramón Areces and the National Marfan Foundation to G.E. O.B. is a recipient of a fellowship from the Ministerio de Economía y Competitividad (Formación de Personal Investigador), and of an EMBO short term fellowship.

\section{Appendix A. Supplementary data}

Supplementary data to this article can be found online at http://dx. doi.org/10.1016/j.yjmcc.2015.05.008.

\section{References}

[1] Marfan A. A case of congenital deformation of the four limbs, more pronounced at the extremities, characterized by elongation of the bones with some degree of thinning. Bull Mem Soc Med Hop Paris 1896;13:220-6.

[2] McKusick VA. The defect in Marfan syndrome. Nature 1991;352:279-81.

[3] Lindsay ME, Dietz HC. Lessons on the pathogenesis of aneurysm from heritable conditions. Nature 2011;473:308-16.

[4] Neptune ER, Frischmeyer PA, Arking DE, Myers L, Bunton TE, Gayraud B, et al. Dysregulation of TGF-beta activation contributes to pathogenesis in Marfan syndrome. Nat Genet 2003;33:407-11.

[5] Doyle JJ, Gerber EE, Dietz HC. Matrix-dependent perturbation of TGFbeta signaling and disease. FEBS Lett 2012;586:2003-15.

[6] Cook JR, Carta L, Galatioto J, Ramirez F. Cardiovascular manifestations in Marfan syndrome and related diseases; multiple genes causing similar phenotypes. Clin Genet 2014:87:11-20.

[7] Dietz HC, Pyeritz RE, Puffenberger EG, Kendzior Jr RJ, Corson GM, Maslen CL, et al. Marfan phenotype variability in a family segregating a missense mutation in the epidermal growth factor-like motif of the fibrillin gene. J Clin Invest 1992;89: $1674-80$.

[8] Summers KM, Raza S, van Nimwegen E, Freeman TC, Hume DA. Co-expression of FBN1 with mesenchyme-specific genes in mouse cell lines: implications for phenotypic variability in Marfan syndrome. Eur J Hum Genet 2010;18:1209-15.

[9] Maki JM. Lysyl oxidases in mammalian development and certain pathological conditions. Histol Histopathol 2009;24:651-60.

[10] Kanematsu Y, Kanematsu M, Kurihara C, Tsou TL, Nuki Y, Liang EI, et al. Pharmacologically induced thoracic and abdominal aortic aneurysms in mice. Hypertension 2010;55:1267-74.

[11] Liu X, Zhao Y, Gao J, Pawlyk B, Starcher B, Spencer JA, et al. Elastic fiber homeostasis requires lysyl oxidase-like 1 protein. Nat Genet 2004;36:178-82.

[12] Maki JM, Rasanen J, Tikkanen H, Sormunen R, Makikallio K, Kivirikko KI, et al. Inactivation of the lysyl oxidase gene Lox leads to aortic aneurysms, cardiovascular dysfunction, and perinatal death in mice. Circulation 2002;106:2503-9.

[13] Remus EW, O'Donnell Jr RE, Rafferty K, Weiss D, Joseph G, Csiszar K, et al. The role of lysyl oxidase family members in the stabilization of abdominal aortic aneurysms. Am J Physiol Heart Circ Physiol 2012;303:H1067-75.

[14] Abraham PA, Perejda AJ, Carnes WH, Uitto J. Marfan syndrome. Demonstration of abnormal elastin in aorta. J Clin Invest 1982;70:1245-52.

[15] Halme T, Vihersaari T, Penttinen R. Lysyl oxidase activity and synthesis of desmosines in cultured human aortic cells and skin fibroblasts: comparison of cell lines from control subjects and patients with the Marfan syndrome or other annulo-aortic ectasia. Scand J Clin Lab Invest 1986;46:31-7.

[16] Yeowell HN, Marshall MK, Walker LC, Ha V, Pinnell SR. Regulation of lysyl oxidase mRNA in dermal fibroblasts from normal donors and patients with inherited connective tissue disorders. Arch Biochem Biophys 1994;308:299-305.

[17] Lindeman JH, Ashcroft BA, Beenakker JW, van Es M, Koekkoek NB, Prins FA, et al. Distinct defects in collagen microarchitecture underlie vessel-wall failure in advanced abdominal aneurysms and aneurysms in Marfan syndrome. Proc Natl Acad Sci U S A 2010;107:862-5.

[18] Judge DP, Biery NJ, Keene DR, Geubtner J, Myers L, Huso DL, et al. Evidence for a critical contribution of haploinsufficiency in the complex pathogenesis of Marfan syndrome. J Clin Invest 2004;114:172-81.

[19] Wilmarth KR, Froines JR. In vitro and in vivo inhibition of lysyl oxidase by aminopropionitriles. J Toxicol Environ Health 1992;37:411-23.

[20] Rabkin SW. Differential expression of MMP-2, MMP-9 and TIMP proteins in thoracic aortic aneurysm-comparison with and without bicuspid aortic valve: a metaanalysis. Vasa 2014;43:433-42.

[21] Pereira L, Lee SY, Gayraud B, Andrikopoulos K, Shapiro SD, Bunton T, et al. Pathogenetic sequence for aneurysm revealed in mice underexpressing fibrillin-1. Proc Natl Acad Sci U S A 1999;96:3819-23.

[22] Cook JR, Carta L, Benard L, Chemaly ER, Chiu E, Rao SK, et al. Abnormal muscle mechanosignaling triggers cardiomyopathy in mice with Marfan syndrome. J Clin Invest 2014;124:1329-39.

[23] Forteza A, Evangelista A, Sanchez V, Teixido G, Garcia D, Sanz P, et al. Study of the efficacy and safety of losartan versus atenolol for aortic dilation in patients with Marfan syndrome. Rev Esp Cardiol 2011;64:492-8.

[24] Groenink M, den Hartog AW, Franken R, Radonic T, de Waard V, Timmermans J, et al. Losartan reduces aortic dilatation rate in adults with Marfan syndrome: a randomized controlled trial. Eur Heart J 2013;39:3491-500.

[25] Habashi JP, Judge DP, Holm TM, Cohn RD, Loeys BL, Cooper TK, et al. Losartan, an AT1 antagonist, prevents aortic aneurysm in a mouse model of Marfan syndrome. Science 2006;312:117-21.

[26] Humphrey JD, Milewicz DM, Tellides G, Schwartz MA. Cell biology. Dysfunctional mechanosensing in aneurysms. Science 2014;344:477-9.

[27] Humphrey JD, Schwartz MA, Tellides G, Milewicz DM. Role of mechanotransduction in vascular biology: focus on thoracic aortic aneurysms and dissections. Circ Res 2015;116:1448-61.

[28] Wagenseil JE, Mecham RP. Vascular extracellular matrix and arterial mechanics. Physiol Rev 2009;89:957-89.

[29] Szauter KM, Cao T, Boyd CD, Csiszar K. Lysyl oxidase in development, aging and pathologies of the skin. Pathol Biol 2005;53:448-56.

[30] Yuan SM, Jing H. Cystic medial necrosis: pathological findings and clinical implications. Rev Bras Cir Cardiovasc 2011;26:107-15.

[31] Yuan SM, Ma HH, Zhang RS, Jing H. Transforming growth factor-beta signaling pathway in Marfan's syndrome: a preliminary histopathological study. Vasa 2011; 40:369-74. 
[32] de Wit A, Vis K, Jeremy RW. Aortic stiffness in heritable aortopathies: relationship to aneurysm growth rate. Heart Lung Circ 2013;22:3-11.

[33] Nollen GJ, Groenink M, Tijssen JG, Van Der Wall EE, Mulder BJ. Aortic stiffness and diameter predict progressive aortic dilatation in patients with Marfan syndrome. Eur Heart J 2004;25:1146-52.

[34] Crosas-Molist E, Meirelles T, Lopez-Luque J, Serra-Peinado C, Selva J, Caja L, et al. Vascular smooth muscle cell phenotypic changes in patients with Marfan syndrome. Arterioscler Thromb Vasc Biol 2015;35:960-72.

[35] Sibon I, Sommer P, Lamaziere JM, Bonnet J. Lysyl oxidase deficiency: a new cause of human arterial dissection. Heart 2005;91:e33.

[36] Majesky MW. Developmental basis of vascular smooth muscle diversity. Arterioscler Thromb Vasc Biol 2007;27:1248-58.

[37] Losenno KL, Goodman RL, Chu MWA. Bicuspid aortic valve disease and ascending aortic aneurysms: gaps in knowledge. Cardiol Res Pract 2012;2012:145202.

[38] Nistri S, Porciani MC, Attanasio M, Abbate R, Gensini GF, Pepe G. Association of Marfan syndrome and bicuspid aortic valve: frequency and outcome. Int J Cardiol 2012;155:324-5

[39] Cook JR, Clayton NP, Carta L, Galatioto J, Chiu E, Smaldone S, et al. Dimorphic effects of transforming growth factor-beta signaling during aortic aneurysm progression in mice suggest a combinatorial therapy for Marfan syndrome. Arterioscler Thromb Vasc Biol 2015;35:911-7.

[40] Atsawasuwan P, Mochida Y, Katafuchi M, Kaku M, Fong KS, Csiszar K, et al. Lysyl oxidase binds transforming growth factor-beta and regulates its signaling via amine oxidase activity. J Biol Chem 2008;283:34229-40.
[41] Kutchuk L, Laitala A, Soueid-Bomgarten S, Shentzer P, Rosendahl AH, Eilot S, et al. Muscle composition is regulated by a Lox-TGFbeta feedback loop. Development 2015;142:983-93.

[42] Rimar D, Rosner I, Nov Y, Slobodin G, Rozenbaum M, Halasz K, et al. Lysyl oxidase is a potential biomarker of fibrosis in systemic sclerosis. Arthritis Rheum 2013;66: 726-30.

[43] Loeys BL, Dietz HC, Braverman AC, Callewaert BL, De Backer J, Devereux RB, et al. The revised Ghent nosology for the Marfan syndrome. J Med Genet 2010:47:476-85.

[44] Holm TM, Habashi JP, Doyle JJ, Bedja D, Chen Y, van Erp C, et al. Noncanonical TGFbeta signaling contributes to aortic aneurysm progression in Marfan syndrome mice. Science 2011;332:358-61.

[45] Junqueira LC, Bignolas G, Brentani RR. Picrosirius staining plus polarization microscopy, a specific method for collagen detection in tissue sections. Histochem J 1979; 11:447-55.

[46] Busnadiego O, Loureiro-Alvarez J, Sandoval P, Lagares D, Dotor J, Perez-Lozano ML, et al. A pathogenetic role for endothelin-1 in peritoneal dialysis-associated fibrosis. J Am Soc Nephrol 2015;26:173-82.

[47] Palamakumbura AH, Trackman PC. A fluorometric assay for detection of lysyl oxidase enzyme activity in biological samples. Anal Biochem 2002;300:245-51.

[48] Wagsater D, Paloschi V, Hanemaaijer R, Hultenby K, Bank RA, Franco-Cereceda A, et al. Impaired collagen biosynthesis and cross-linking in aorta of patients with bicuspid aortic valve. J Am Heart Assoc 2013;2:e000034. 